\title{
A Piezoresistive MEMS Sensor for Acoustic Noise Measurements
}

\author{
A. Naguib* \\ Michigan State University, East Lansing, MI \\ E. Soupos and H. Nagib ${ }^{\dagger}$ \\ Illinois Institute of Technology, Chicago, IL \\ C. Huang and K. Najafi \\ University of Michigan, Ann Arbor, MI
}

\begin{abstract}
A MEMS piezoresistive acoustic sensor has been developed for measurement of jet screech noise. The new sensor was calibrated in the sound field of an air siren. Two sensors with a size of 510 and $710 \mu \mathrm{m}$ were tested and compared to commercial sensors. The results show that the MEMS sensors are five to eight times more sensitive than the smallest commercially available piezoresistive sensors. Furthermore, the sensors are stable as demonstrated by the agreement between calibrations conducted over two months period. However, the bandwidth of this first generation sensors is somewhat limited by the damping of the diaphragm motion. This effect will be remedied in the next generation sensors through careful minimization of damping effects and increase of the sensors natural frequency.
\end{abstract}

\section{Introduction}

Background

Over the past decade there has been a growing interest in exploring the ability of Micro Electro Mechanical Systems (MEMS) technology to provide sensors, actuators and ultimately, systems suitable for use in control and diagnostics in aerospace applications. This interest in MEMS stems from various reasons. First, the ability of the technology to construct

* Member AIAA, Assistant Professor

${ }^{\dagger}$ Senior Member AIAA, Associate Professor

Copyright $\bigcirc$ The American Institute of Aeronautics and Astronautics Inc. All right reserved conventional sensors at a scale that is one to two orders of magnitude smaller than currently available results in previously unachievable temporal and spatial resolutions. Second, the ability of MEMS to produce dense sensor arrays, integrated with actuators and electric circuitry, has the potential for producing small, lightweight, low-power, autonomous control systems for a wide range of applications.

Albeit the list of advantages of MEMS, the technology is currently at its frontiers and answers to various questions regarding the technology and its utility are currently being researched. Some of the more important questions concern device characteristics, performance in the application environment and packaging of devices and systems. For an overview of MEMS and its aerospace and fluid mechanics applications, see Ho et al. ${ }^{1}$

Perhaps one of the most important applications of MEMS in fluid mechanics diagnostics is in conducting time- and space-resolved measurements of the surface pressure. Such measurements would be useful for understanding flow-induced noise and vibrations in aeroacoustic problems. For example, the turbulent wall pressure fluctuations caused by the boundary layer flow structure over an airplane fuselage generate undesired cabin noise. Complete characterization of the wall-pressure frequency/wavenumber excitation of 
the fuselage at the high Reynolds numbers encountered during flight requires a large array of small (less than $100 \mu \bar{m})$ pressure sensors. Achievement of such an array may only be possible through MEMS. Other applications of acquiring timeresolved surface pressure maps include, but are not limited to, phased sensor array for localization of sound sources and active control of fan noise in turbine engines.

Dynamic measurements using MEMS-based piezoresistive pressure sensors have been conducted by Löfdahl et al. ${ }^{2}$ and Sheplak et al. ${ }^{3}$ The piezoresistive sensor from Löfdahl et al. ${ }^{2}$, was constructed using a $0.4 \mu \mathrm{m}$-thin polysilicon diaphragm. The deflection of the diaphragm was measured using a polysilicon piezoresistor deposited on top of the $100 \times 100 \mu^{2}$ diaphragm. The sensor's static and acoustic sensitivities were determined to be $0.12 \mu \mathrm{V} / \mathrm{V} \mathrm{Pa}$ and $0.09 \mu \mathrm{V} / \mathrm{V} \mathrm{Pa}$, respectively. The acoustic response was uniform to within $+/-3 \mathrm{~dB}$ from $10 \mathrm{~Hz}$ to $10 \mathrm{kHz}$. Löfdahl et al. ${ }^{2}$ demonstrated the utility of their sensor by conducting measurements beneath a turbulent boundary layer using an array of six pressure sensors.

At MIT, Sheplak et al. ${ }^{3}$ constructed a silicon-based microphone with a piezoresistive sensing scheme for use in wind tunnel tests in NASA's High-Speed Civil Transport program. The primary sensing element of the microphone was a $1.5 \mu \mathrm{m}$-thick, $210 \mu \mathrm{m}$-diameter silicon-nitride membrane. On top of the membrane, single-crystal silicon piezoresistors were used in half or full bridge configuration for detection of the diaphragm strain under the action of the measured sound-field pressure. A $10 \mu \mathrm{m} \times 10 \mu \mathrm{m} \times 2.25 \mathrm{~mm}$ long channel provided static pressure equalization for the microphone. Sheplak et al. ${ }^{3}$ pointed out that the use of single-crystal silicon for construction of the piezoresistors resulted in about five times enhancement in the sensor sensitivity over that of commercial sensors with similar construction and sensing scheme. The sensitivity of the microphone was $2.2 \mu \mathrm{V} / \mathrm{V} \mathrm{Pa}$ and was flat, to within $3 \mathrm{~dB}$, from $200 \mathrm{~Hz}$ up to at least $6 \mathrm{kHz}$.

Preliminary characterization of the MEMS sensors discussed in the current work was recently presented by Naguib et $\mathrm{al}^{4}$. Two sensor sizes were investigated: 510 and $710 \mu \mathrm{m}$. The results, which were confined to a frequency range of $1.5-5 \mathrm{kHz}$, showed that the frequency response was flat to within $1 \mathrm{~dB}$. The sensitivity of the $510 \mu \mathrm{m}$ sensor was similar to that of a commercial Kulite XCS- 062 probe. On the other hand, the $710-\mu \mathrm{m}$ sensor had a sensitivity that was three to four times larger than the Kulite sensor. In this investigation, the characterization results of the MEMS sensor are extended to cover a frequency range of $100 \mathrm{~Hz}-10 \mathrm{kHz}$. The results provide better understanding of the dynamic behavior of the sensors.

\section{Construction and fabrication of the current MEMS}

\section{sensor}

The MEMS sensor used in the current investigation has been developed at the University of Michigan for use as part of an array to measure the sound field at the lip of an axi-symmetric jet during supersonic jet screech. Ultimately, the acoustic sensors, integrated with MEMS actuators are to be used to implement a feedback based control algorithm aimed at reduction/cancellation of screech noise. Development and testing of the actuators are not the subject of this paper.

The MEMS acoustic sensor consists of a stresscompensated PECVD silicon nitride/oxide, $0.4 \mu \mathrm{m}$-thick diaphragm together with four mono-crystalline ionimplanted $\mathrm{p}^{++}$silicon piezoesistors. The coefficient, $\pi_{44}$, for this type of piezoresistors is about four times larger than that based on p-type polysilicon; thus, leading to a higher transducer sensitivity. The piezoresistors are arranged in a full Wheatstone-bridge configuration for detection of the diaphragm deflection. Two of the lead wires connected to the four corners of the bridge are used to provide $10 \mathrm{~V}$ excitation to the bridge. The remaining two wires carry the differential output signal of the bridge which is proportional to the measured pressure.

Figure 1 (top) shows a SEM view of the pressure sensor, with a close-up view of one of the piezoresistors. Figure 1 (bottom) displays two of the sensors integrated with two actuators. The piezoresistive readout scheme for the sound detector is chosen in this research because of several reasons:

1. The sound level is high enough ( $>100 \mathrm{~dB}$ SPL) that the slightly lower sensitivity of a piezoresistive readout does not limit the performance.

2. The fabrication and readout of a piezoresistive sound detector are much simpler than either a piezoelectric or capacitive microphone.

3. The bandwidth of a piezoresistive device is not as affected by air damping typically encountered in a capacitive device with a small air gap.

A brief outline of the manufacturing process of the acoustic sensors is provided in Figure 2. For a more detailed description of sensor manufacturing, see Huang et $a l^{5}$ 
Two sensors were tested in this study. The diaphragm size for the two sensors was $510 \times 510 \mu \mathrm{m}^{2}$ and $710 \mathrm{x}$ $710 \mu \mathrm{m}^{2}$. The sensors, which were on the same chip, had a nominal piezoresistor values of 4 and $6 \mathrm{k} \Omega$, respectively.

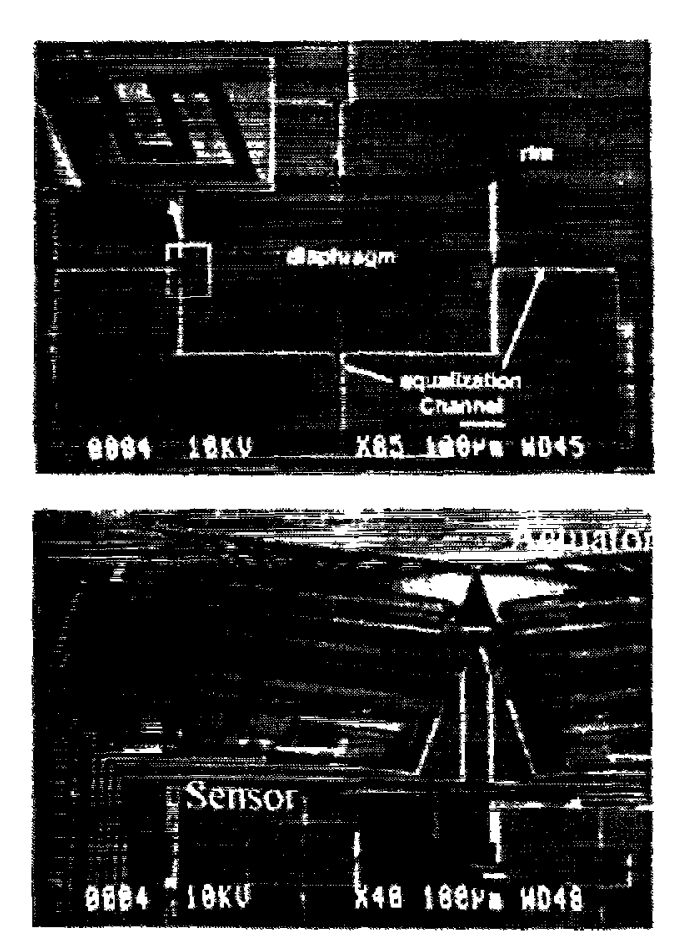

Figure 1. SEM Views of the Acoustic Sensor (top) and Integrated Sensor/Actuator System (bottom)

\section{Experimental Setup and Data Analysis}

Since the MEMS sensor was designed for screech noise measurements, it was desired to calibrate the sensor in a an acoustic field at a sound pressure level (SPL) of at least $100 \mathrm{~dB}$ over a wide frequency. To generate this sound field it was not possible to use a speaker due to the contamination of the MEMS output signal with noise from the audio amplifier used to drive the speaker. This was believed to be due to the lack of appropriate shielding and grounding of the MEMS sensor wiring. Therefore, it was decided to generate the sound field via non-electrical means. To this end, an "air siren" was constructed. The siren consisted of a $6 \mathrm{~mm}$-diameter air jet that was "shuttered" periodically using a $0.15-\mathrm{m}$ diameter chopper wheel with slots cut along the circumference of the wheel. The passage of the slots in front of the jet created pressure pulsation at the slotpassing frequency, thus generating sound at a frequency that was adjustable by changing the disc rotation speed.
The minimum and maximum limits on the disc rotation rate were determined, respectively, by the lowest stable rotational speed and maximum driving voltage of the DC electric motor used to rotate the chopper disc. The corresponding frequency limits of the generated sound were in the range of $1.5-5.5 \mathrm{kHz}$ when using a fifty-slot disc. To cover the full range of $100 \mathrm{~Hz}-10 \mathrm{kHz}$, four discs were used with two, ten, fifty and one hundred slots.

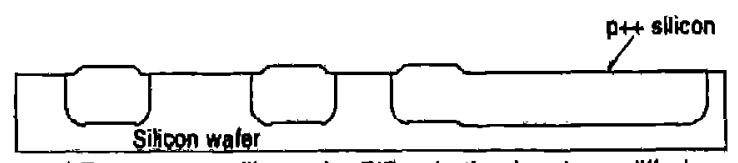

b) Etch recess In silicon using RIE; selective deop boron diffusion

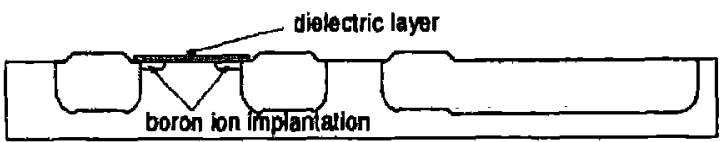

b) Selective boron ion implantation; RTA; LTO; PECVD

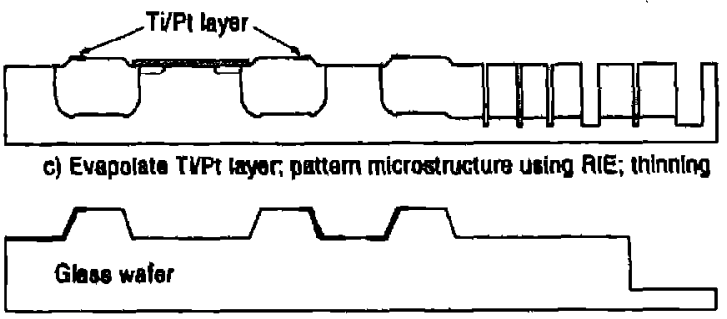

d) Glase rocess; evaporate metal; groove glase

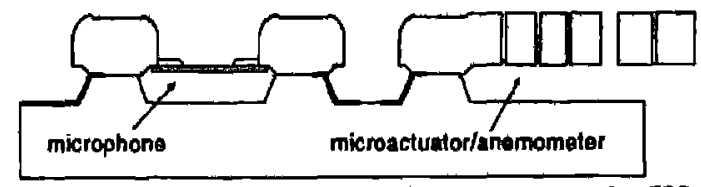

o) Electrostatically bond silicon to the paltem glase waler; EDP

Figure 2. The Fabrication Process of the MEMS Sensor/Actuator System

The location of sensor calibration within the siren's sound field was chosen to be outside the boundaries of the air jet flow. This was done to insure that the output of the sensor was due to the acoustic and not the hydrodynamic pressure fluctuations. The resulting sensor location was about $0.6-\mathrm{m}$ away from the jet exit at an angle of $45^{\circ}$ from the jet axis, as seen in Figure 3. The SPL at the measurement location was measured as a function of sound frequency using an $1 / 8$ " Bruel \& Kaejer microphone. A sample of the results is displayed in Figure 4 for the fifty-slot disc. As seen from Figure 4, large sound levels of 135-145 dB were achieved over the frequency range from 2 to $5.5 \mathrm{kHz}$ at the calibration location. 
To obtain the MEMS sensor's dynamic response, the sensor was placed next to a commercial pressure transducer with known characteristics in the sound field of the siren at the calibration location. The 'reference' transducer was a Kulite model XCS-062-5G with a nominal sensitivity of $0.2 \mu \mathrm{V} / \mathrm{V} \mathrm{Pa}$ and a flat frequency response up to about $20 \mathrm{kHz}$. Although larger in size, the Kulite sensor also utilizes a silicon diaphragm with four piezoresistors arranged in a Wheatstone bridge to detect the deflection of the diaphragm under the action of the measured pressure.

Due to the similar principle of operation of the MEMS and the Kulite sensors, the excitation voltage and signal conditioning of the Kulite and MEMS sensors were achieved using AD 1B31AN strain gage signal conditioner from Analog Devices. The Kulite output was amplified by a factor of 2500 using the signal conditioner, while the MEMS sensor signal was amplified by a factor of 2000 . - The signals were further low-pass filtered at the Nyquist frequency to eliminate eliasing during data recording.

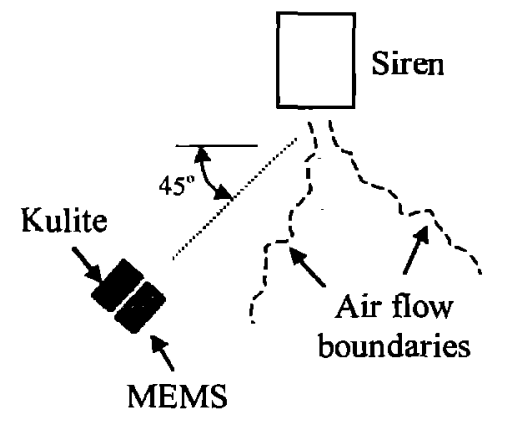

Figure 3. A Schematic of the Calibration Setup

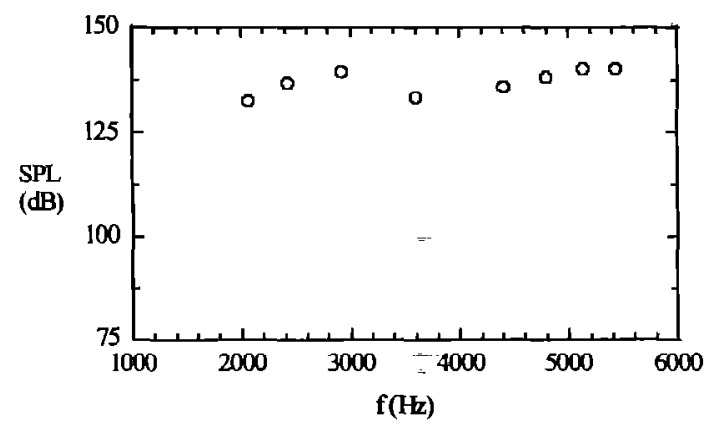

Figure 4. Siren Sound Pressure Level at Calibration Location

The output of the Kulite and MEMS sensors were acquired simultaneously at a sampling rate of ten times the sound frequency. The measurements were used to obtain the power spectra of the MEMS and Kulite voltage time series. 400 records of 2048 points were used to obtain each spectrum. This resulted in a spectrum frequency resolution of $0.5 \%$ of the sound frequency. The random uncertainty in the spectral estimate was approximately $5 \%$ based on 400 records and assuming Gaussian random variation in the measurements. The sensitivity of the MEMS sensor at a given frequency was determined from the equation:

$$
K_{M E M S}=K_{\text {Kulite }} \sqrt{\frac{E_{v v, M E M S}}{E_{v, \text { Kulite }}}},
$$

where, $K_{\text {Kulite }}$ is the Kulite sensitivity in $\mathrm{mV} / \mathrm{Pa}$ and $E_{v v, M F M S}$ and $E_{v v, \text { Kulite }}$ represent the energy contained in the voltage spectrum peak at the frequency of the siren sound for the MEMS and Kulite, respectively. The energy values were obtained from integration of the spectra over a narrow frequency range around the sound frequency. The integration was necessary due to some jitter in the motor rotational speed observed during data recording. This jitter, however, was less than $4 \%$ for all frequencies.

\section{Results and Discussion}

Prior to calibration of the MEMS sensors in the siren's sound field, it was desired to verify the calibration process. Therefore, a $1 / 8$ " B\&K microphone with known sensitivity was used in place of the MEMS sensor and calibrated against the Kulite as outlined in the previous section. The results of the calibration are compared to the $\mathrm{B} \& \mathrm{~K}$ calibration provided by the manufacturer in Figure 5. The broken lines show the manufacturer provided calibration along with a $\pm 3 \mathrm{~dB}$ tolerance band. As seen from the figure, the calibration procedure used here captures the actual $B \& K$ response to within $\pm 3 \mathrm{~dB}$. Although $\pm 3 \mathrm{~dB}$ error may not be good enough for conducting accurate measurements, it should be sufficient for providing general characterization of the response of the MEMS sensors. It should be mentioned here that the $B \& K$ phase response was also checked. These results, however, were largely scattered and inconsistent with the known phase response of the B\&K microphone. Therefore, no calibration results will be provided for the MEMS phase characteristics.

Another important check on the MEMS calibration procedure is to verify that the MEMS output due to the acoustic measurements is much larger than the electrical noise level. To achieve this, the spectra measured by the MEMS, with and without the acoustic field, were compared. It was possible to turn the acoustic field off by simply shutting down the air supply to the siren while 
the motor is running. A sample of the results at a motor rotation speed of 6000 RPM is shown in Figure 6. Inspection of the figure shows that while the air and motor are turned on, a large acoustic peak exists at 5.5 $\mathrm{kHz}$. The magnitude of this peak is at least four orders of magnitude larger than the background noise observed when shutting the air supply only, or when shutting off both the air supply and the motor. This also demonstrates that the peak in the spectrum at the acoustic frequency is in fact due to the acoustic field, and not due to any electrical noise induced by the motor. As mentioned earlier, induced electrical noise was a problem when attempting to use an audio amplifier coupled to a speaker to conduct the calibration.

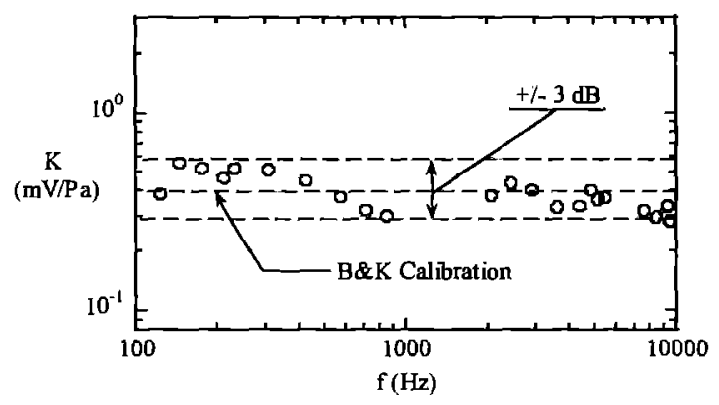

Figure 5. Response Check of the B\&K Microphone

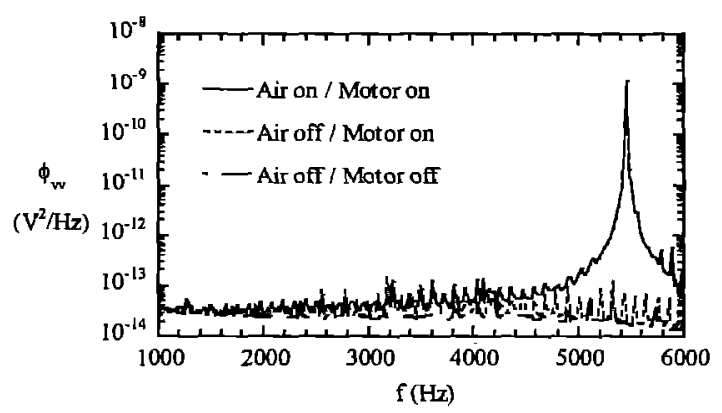

Figure 6. Signal to Noise Level at Calibration Location

The voltage spectra measured using the $510-\mu \mathrm{m}$ and $710-\mu \mathrm{m}$ sensors for three different rotational speeds of the siren are provided in Figures 7 and 8 . For comparison purpose, the pressure spectra measured using the Kulite under the same identical conditions as the MEMS are also provided in the Figures. It is seen from Figures 7 and 8 that the shape of the spectrum obtained from the MEMS and Kulite sensors seems to agree well for both sensor sizes. It should be noted that the height of the spectrum peak relative to the background noise (i.e., the flat part of the spectrum) may be used to assess the signal to noise ratio of the sensors. Inspection of
Figures 7 shows that this peak height above the noise level is similar between the $510-\mu \mathrm{m}$ and Kulite sensors for the frequency range between 1 and $5 \mathrm{kHz}$. For the same frequency range, the $710-\mu \mathrm{m}$ sensor seems to have a signal to noise ratio that is about two to three times better than the Kulite (see Figure 8 ).

Figure 9 provides the full dynamic calibration results for the MEMS sensors. For reference, the sensitivity of the Kulite and B\&K microphone is also provided in Figure 9. The open squares and closed circles provide the current calibration results for the $510-\mu \mathrm{m}$ and $710-\mu \mathrm{m}$ sensors, respectively. The rest of the symbols provide calibration results obtained for the same sensors twomonths earlier over a narrower frequency range. Also, note that the error bars shown in the figure represent the calibration procedure's $\pm 3 \mathrm{~dB}$ uncertainty discussed earlier.

The results in Figure 9 demonstrate that a very good agreement is obtained between calibrations conducted two-months apart. This agreement indicates that the MEMS transducers tested here are very stable and reliable sensors. For frequencies below $1 \mathrm{kHz}$, the sensitivity of the MEMS sensors seems to be almost an order of magnitude larger than the sensitivity of the commercial Kulite sensor. At higher frequencies, however, the MEMS sensitivity is attenuated. This attenuation is more pronounced for the response of the $510 \mu \mathrm{m}$ sensor which drops to the same level as that of the Kulite.

To better characterize the results obtained in Figure 9, it is useful to consider the electrical analog circuit of the MEMS sensor (Rossi ${ }^{6}$ ). This electric circuit analogy, which is based on the assumption of lumped system parameters, can be used to derive an expression for the response of the MEMS sensor. The sensor response predicted in this fashion should be reasonably accurate over the frequency range investigated since the corresponding wavelength of sound is considerably larger than the sensor size. Figure 10 shows the electric circuit analog of the MEMS sensor. In this figure, $\mathrm{L}_{\text {vent, }}$, $L_{r}$ and $L_{d}$ represent the mass of the air in the vent volume, the radiation mass and the diaphragm mass, $R_{\text {vent }}, R_{r}$ and $R_{\text {gap }}$ represent the dissipation of acoustic energy in the vent, by sound radiation and in the gap behind the diaphragm, and $C_{d}$ and $C_{c}$ represent the compliance of the diaphragm and the cavity, or air gap, behind the diaphragm, respectively.

The analysis based on the equivalent circuit in Figure 10 predicts that the sensor response above a low cut-off frequency (of the order of tens of $\mathrm{Hz}$ ) is simply represented by a second order system. Consequently, a 
curve representing the response of a second order system was fitted to the results in Figure 9 for the 510 and 710 $\mu \mathrm{m}$ sensors. The outcome of the fitting process is given in Figure 11. Since an equally good fit was obtained with different combinations of the natural frequency $\left(f_{n}\right)$ and damping ratio $(\zeta)$ of the second order response, three different curve fits are provided in Figure 11. Those fits correspond to natural frequencies of 5,10 and $100 \mathrm{kHz}$ and are shown in the top, middle and bottom plots in Figure 11, respectively.
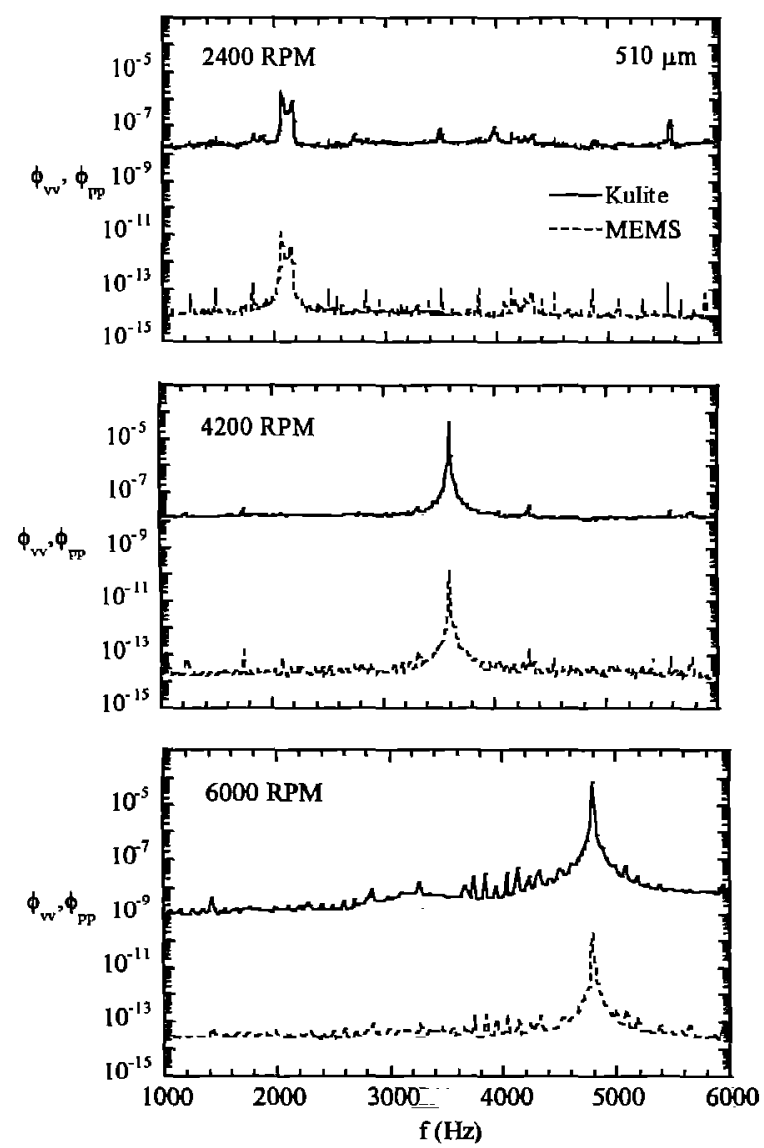

Figure 7. Spectra Measured by the $510 \mu \mathrm{m}$ MEMS Sensor and Kulite

Using the curve fitting results, the "static" sensitivity of the MEMS sensors was estimated to be $0.012 \mathrm{mV} / \mathrm{Pa}$ and $0.018 \mathrm{mV} / \mathrm{Pa}$ for the 510 and $710-\mu \mathrm{m}$ sensors, respectively. Remarkably, those sensitivity values are five to eight times larger than that of a Kulite XCS-062$5 \mathrm{G}$ sensor. The bandwidth, of the MEMS sensors, however, is smaller than that of the Kulite sensor. This is due to the attenuation seen in the response curves in Figure 11. The response attenuation is related to the damping of the diaphragm motion, which in turn is dependent on $R_{\text {gap }}$ in the equivalent circuit in Figure 10. This gap resistance is primarily caused by viscous dissipation of the air motion inside the cavity behind the diaphragm. Since the cavity volume is smaller for the smaller sensor, more damping effects are expected for the $510-\mu \mathrm{m}$ than for the $710-\mu \mathrm{m}$ sensor. This agrees with the more substantial attenuation observed for the 510- $\mu \mathrm{m}$ sensor.
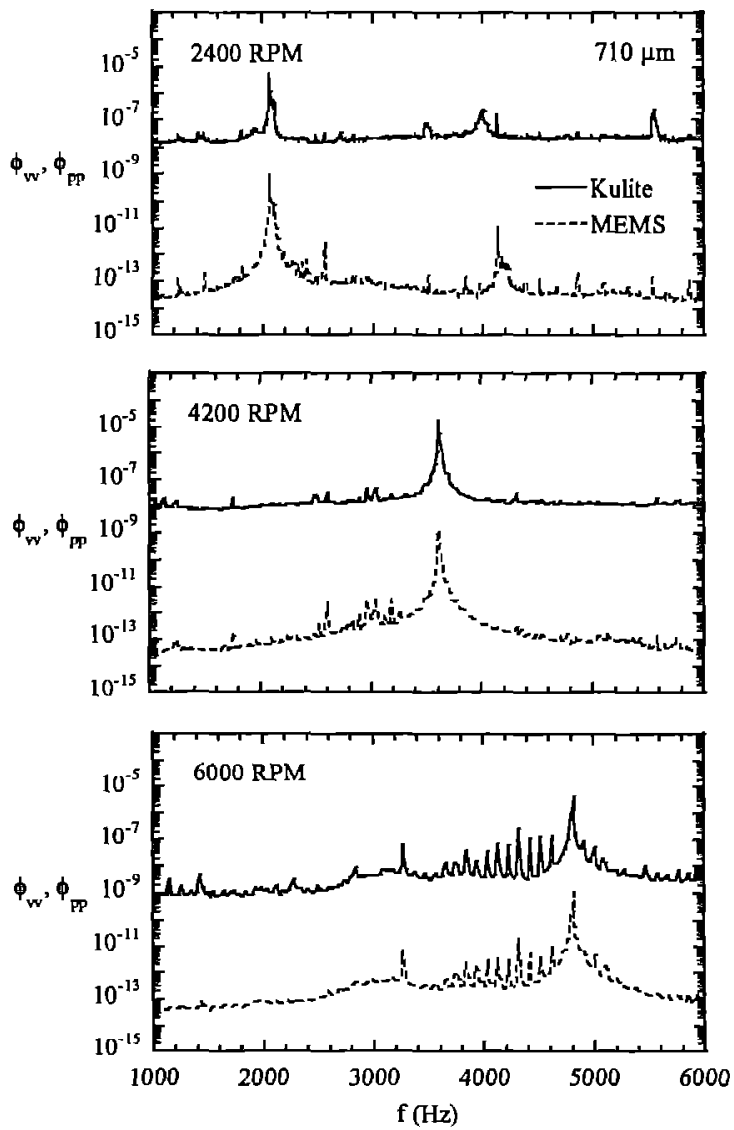

Figure 8. Spectra Measured by the $710 \mu \mathrm{m}$ MEMS Scnsor and Kulite

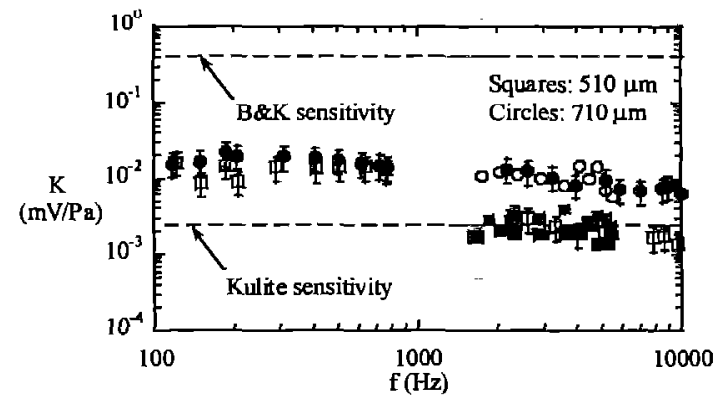

Figure 9. Response of the MEMS Sensors Compared to the Kulite and $B \& K$ 


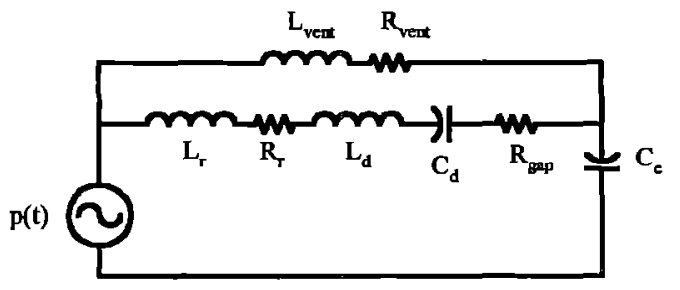

Figure 10. Equivalent Circuit of MEMS Sensor
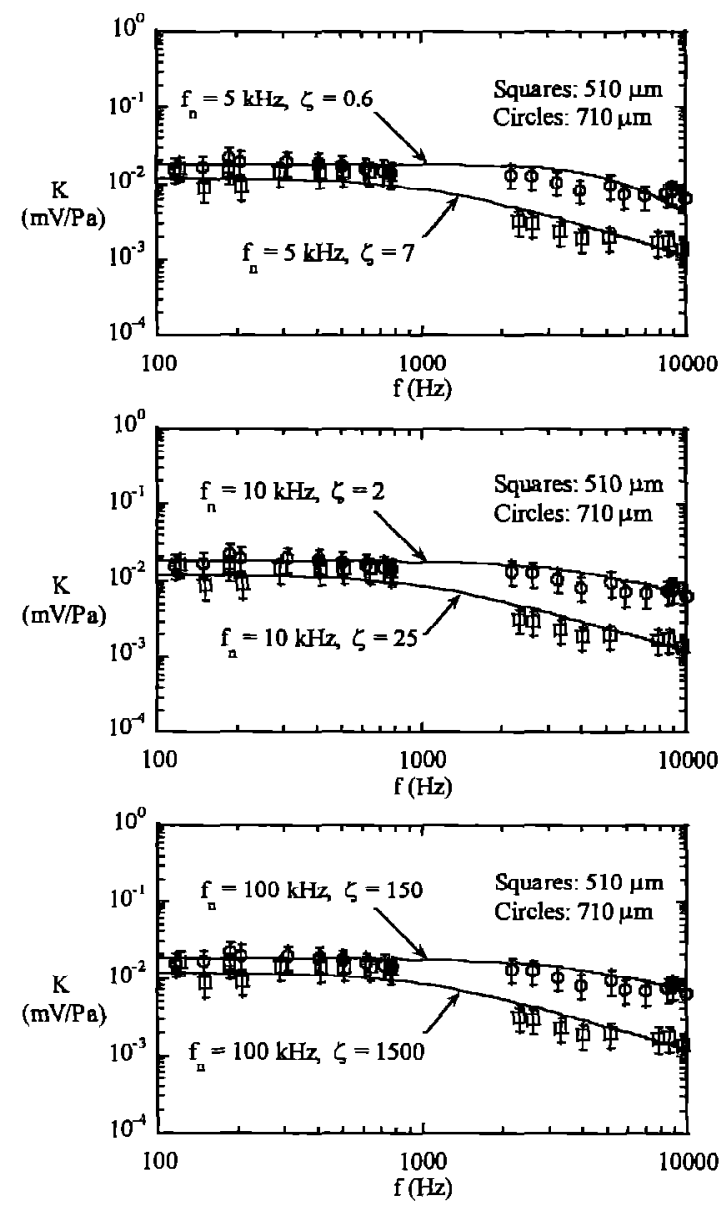

Figure 11. Comparison of the Response of the MEMS Sensors to $2^{\text {nd }}$-Order System Response.

Finally, it should be mentioned here that it is difficult to believe that the damping ratio for the MEMS sensors is of the order of 100 or more. Therefore, it is more likely that the MEMS response is characterized by the curve fits in the top or middle portion of Figure 11. That is, the natural frequency of the sensors is likely to be in the $5-10 \mathrm{kHz}$ range with a damping factor that is larger than 0.6 but less than 10 . Those findings suggest that in the sccond-generation devices, more attention should be given to understand and minimize the diaphragm motion damping. Additionally, the natural frequency should be enhanced to be of the order of $100 \mathrm{kHz}$ or more. This should not be difficult to achieve for sensors as small as those investigated here.

\section{Conclusions}

A new type of MEMS piezoresistive acoustic sensor has been developed and tested in the sound field of a siren. Test results showed that the sensitivity of the new sensors was about five to eight times the sensitivity of the commercial silicon-based Kulite scnsor. Moreover, repeated calibrations of the sensor over a two-month period demonstrated that the new sensors are highly stable. On the other hand, comparison of the sensor dynamic response to that of second order systems suggested that the damping of the diaphragm motion results in undesired attenuation of the sensor response. Future generations of the new device will be designed such as to minimize the damping effects and increase the natural frequency.

\section{Acknowledgement}

This work was sponsored by the Air Force Office of Scientific Research, USAF, under grant/contract number F49620-96-1-0459.

\section{References}

${ }^{1}$ Ho, C. M., Tung, S., Lee, G. B., Tai, Y. C., Jiang, F. and Tsao, T., "MEMS- A Technology for Advancements in Aerospace Engineering", American Institute of Aeronautics and Astronautics, Paper 97-0545, 1997.

${ }^{2}$ Löfdahl, L., Kälvesten, E. and Stemme, G., "Small Silicon Pressure Transducers for the Space-Time Correlation Measurements in a Flat Plate Boundary Layer," FED-Vol. 197, Application of Microfabrication to Fluid Mechanics, ASME 1994.

3 Sheplak, M., Breuer, K.S., \& Schmidt, M.A. "A wafer-bonded, silicon-nitride membrane microphone with dielectrically-isolated, single-crystal silicon piezoresistors." Proceedings of the Solid-State Sensor and Actuator Workshop, Hilton Head, SC, June 1998.

${ }^{4}$ Naguib, A., Soupos, E., Nagib, A., Najafi, K. and Huang, C., "Characterization of a MEMS Acoustic/ Pressure Sensor ", American Institute of Aeronautics and Astronautics, Paper 99-0520, 1999.

5 Huang, C., Najafi, K., Alnajjar, E., Christophorou, C., Naguib, A. and Nagib, H., "Operation and Testing of Electrostatic Microactuators and 
Micromachined Sound Detectors for Active Control of High Speed Flows," Proceedings, IEEE/ASME Micro Electro Mechanical Systems Workshop, Heidelberg, Germany, January 1998.

'Rossi, M. "Acoustics and Electroacoustics," Artech House, Norwood, MA, 1988. 